\title{
Correspondence not yet for closing?
}

\author{
Special relativity is the most familiar graveyard of general understanding. But can general understand- \\ ing be advanced when discussion of particular points is suspended?
}

WHEN can a controversial topic fairly be held to have run its course? This question arises in the management of any correspondence column even when the contributors are as uniformly discerning, and as free from the temptation to grind parochial axes, as those who are good enough occasionally to write to this journal. Sometimes, it seems that correspondents have said everything it is possible to say about a topic, that the chance that one or other of two disputant sides will change its mind has shrunk to zero or that people are about to move from intellectual argument to personal abuse. Then, editors feel free to wash their hands of the business with the somewhat pompous declaration "This correspondence is now closed".

Just that has just happened in Physics Bulletin, the monthly news journal of the British Institute of Physics, which in February stumbled into what promised to be an entertaining and even instructive vein of correspondence by publishing a brief article "Energy has mass" by Sir Hermann Bondi and C.B. Spurgin (Phys. Bull. 38, 62; 1987). The declared objective was to rid special relativity of a "common misunderstanding". The next step, in April, was a letter of dissent from Sir Rudolph Peierls (ibid.p.127) which must have excited readers to look forward to fireworks ahead.

In the event, the outcome is anticlimax; Norman Dombey, Sir William McCrea and John Rousseau say at some length that Bondi and Spurgin have not dispelled confusion, but have added to it, Bondi and Spurgin confess that that may indeed have been the case and the editor of Physics Bulletin says that the correspondence "has now been closed". The past tense suggests a greater degree of finality than usual, even a greater of degree of finality than readers of the journal will welcome.

What can be the hare run so quickly to ground? Citing half a dozen textbooks and a schools examination question to illustrate what they describe as "the serious nature of this misunderstanding", Bondi and Spurgin originally set out to show that most people misinterpret Einstein's equation $E=m c^{2}$ as a proof that "mass and energy are interconvertible". One of the worst offenders is the Smyth Report, the document that in 1946 provided the first rattling account of the way in which nuclear fission functions, but which rested much of its case on the assumption that mass can be sometimes turned into energy. This, Bondi and Spurgin said, is not the case. On the contrary, energy and mass are governed by separate conservation laws which ensure that the amounts of energy and of mass in a physical system are strictly constant. Specifically, "students should be taught that:

(1) energy has mass;

(2) energy is always conserved;

(3) mass is always conserved".

What Einstein's equation shows, Bondi and Spurgin say, is merely that energy has mass, or inertia, a point they illustrate by calculating the difference between the mass of a moving particle and its restmass, which is the relativistic kinetic energy divided by $c^{2}$, or $1 / 2 m c^{2}$ when $v$, the velocity, is small compared with $c$, the velocity of light. Another way of putting this is to observe that a photon, which has zero rest-mass, plainly has inertia because, when stopped, it can transfer momentum to an atom.

So how did misunderstanding come to pervert discussion of Einstein's equation? Bondi and Spurgin said that "simplified popular accounts of nuclear fission processes" may have been responsible. True, they say, the combined mass of a ${ }^{235} \mathrm{U}$ nucleus and a neutron (needed to stimulate fission) is greater than that of the products, while "a very considerable amount of energy seems to have appeared from nowhere". But those who have been putting about the heresy that this is an illustration of the conversion of mass into energy have either forgotten or malevolently concealed the circumstance that energy has mass $E / c^{2}$ which, when added to the combined mass of the fission products, saves the appearance and the integrity of the principle of the conservation of mass.

Peierls' comment is the more direct, amounting merely to the assertion that it is only a matter of convention, or of convenience, whether the kinetic energy of the fission fragments (which becomes the heat produced by nuclear reactors) is counted in Einstein's equation as mass or as energy.

The difficult case is that of the annihilation of a positron and an electron, most simply supposed at rest. The end-product consists only of photons of zero rest-mass. Would it be more appropriate to say that the final state has energy, or that it has mass (which is equal to the measurable energy of the photons divided by $c^{2}$ )?

Dombey, McCrae and Rousseau go further, pointing out that the tangent vector to the world-line of a particle by which its evolutionary history is described, often called the momentum fourvector or the four-momentum, has a fixed length equal to the rest mass (multiplied by $c^{2}$ ), that the time-like component of the four-momentum (for a particle, the mechanical energy) depends on the reference frame and on its velocity but that the mechanical energy of a system of immutable particles is conserved within a single frame of reference, and so on. Concerning the question of the annihilation of positrons and electrons, they ask directly "why Bondi and Spurgin protest at this being described as the conversion of mass into energy".

To be fair to everybody, the answer they get is not particularly satisfactory. Bondi and Spurgin, while acknowledging that they "may have started more of a discussion, with more of a danger of confusion, than we had realised", go on to say that the differences between them and their critics "are differences in terminology and the perceived needs of readers". They say that they still believe their quotations from other people's textbooks "were only too liable to create a wrong understanding" and that their separate listing of the conservation laws of mass and energy "no more meant they were separate" than "stating such a law in English and in French means that there are two separate laws".

So, in the end, it seems that everybody is at one. "If we wrote the article again", it would have been emphasized that "mass" means nothing but "measure of inertia". No doubt, the authors would also have taken greater care to rid their text of emotive headlines such as "exploding myths" and statements that students should be "urged to avoid such terminology as "the equivalence of mass and energy". In any case, the upshot is that there is only one conservation law, although it is far from clear whether people "to whom the distinction between rest mass and inertial mass is less obvious than it is to, say, particle theorists" can be trusted with that knowledge. "We can only ... hope that ... the clarity will result that we are all so keen to create."

With such a goal, how could such a correspondence be closed or, worse, "have been closed". John Maddox 